\title{
diffusion-îurdarrentals
}

The Open-Access Journal for the Basic Principles of Diffusion Theory, Experiment and Application

\section{Analytical solution for the time dependent self-diffusion coefficient of a liquid in a porous medium}

\author{
Valentin Loskutov, Vyacheslav Sevriugin \\ Mari State Pedagogical Institute, Physics and Mathematics Dept.,Yoskar-Ola, Russia \\ Corresponding author: 424002, Loskutov Valentin, Physics and Mathematics Dept., Mari \\ State Pedagogical Institute, Kommunisticheskaya st., 44, Yoshkar-Ola, Mari El Republic, \\ Russia \\ E-Mail: val_losk@rambler.ru
}

(received 13 May 2007, accepted 7 June 2007)

\begin{abstract}
The purpose of our work is to attempt to find the analytical expression approximating the experimentally obtained $D(t)$ dependence of molecules of liquids or gases, in porous systems. The statement of the problem is based on the most general representations of selfdiffusion processes in porous systems.
\end{abstract}

\section{Keywords}

porous media, self-diffusion, time dependence

\section{Introduction}

The time dependence of the self-diffusion coefficient $D(t)$ for a liquid molecule in a porous medium, obtained in NMR-PFG experiments, gives important information on features of diffusion processes in the porous systems investigated. Now there is great volume of experimentally obtained time dependence of $D(t)$ over wide ranges of diffusion times for various liquids in natural and model porous systems. However, the problem of interpretation still remains. Besides it is necessary to note, that the description of dependence of selfdiffusion coefficient from media geometry and features of «porous media - liquid» interaction in the total interval of diffusion times is absent.

The purpose of our work is to attempt to find the analytical expression approximating the experimentally obtained $D(t)$ dependence of molecules of liquids or gases, in porous systems. The statement of the problem is based on the most general representations of selfdiffusion processes in porous systems. 


\section{Theory}

For any diffusion regime the result of NMR diffusion experiment represents a diffusion attenuation of a signal spin echo $\Psi(\vec{q}, t)$, which in the most general case can be written as

$$
\Psi(\vec{q}, t)=\int F(\vec{R}, t) \exp (i \vec{q} \cdot \vec{R}) d \vec{R},
$$

where $F(\vec{R}, t)$ is function of probability distribution density for diffusion displacements $R$ of molecules during exposition time $t, \vec{q}=\gamma \vec{g} \delta, \gamma$ is the gyromagnetic ratio, $g \delta$ the operating size of a pulse gradient of a magnetic field with magnitude $g$ and duration $\delta$. In traditional diffusion experiment [1] a gradient of a magnetic field is assumed to be directed on a direction $\vec{x}$ of an external magnetic field. In this case Eq.(1) can be written in the form:

$$
\Psi\left(q_{x}, t\right)=\int F(x, t) \cos \left(q_{x} \cdot x\right) d x .
$$

If the functions $F(R, t)$ and $F(x, t)$ are given by a Gaussian distribution, the diffusion attenuation (2) is an exponential dependence of the kind

$$
\Psi(q, t)=\exp \left(-q^{2} D t\right)
$$

where $D$ is the self-diffusion coefficient of a diffusant molecule. In case of a nongaussian function $F(R, t)$ the diffusion attenuation $\Psi(\vec{q}, t)$ is no exponential function and the interpretation has to be based on additional information on investigated system.

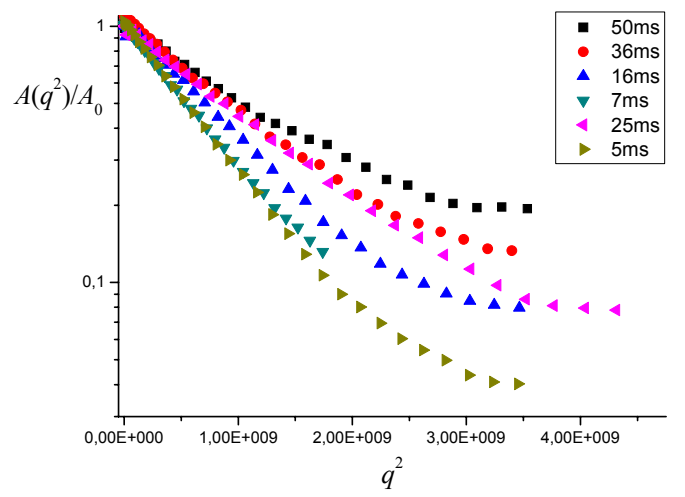

Fig. 1: Diffusion attenuation of spin echo amplitude, received in NMR PFG experiment for the benzene entering porous medium, formed by packed glass spheres with diameter $53 \div 63 \mu \mathrm{m}$, for various diffusion times.

The nonexponentiality of the diffusion attenuations of a spin echo signal is a typical feature of the NMR diffusion experiment in porous media. As a typical example, fig. 1 presents diffusion attenuations of the spin echo signal received by NMR PFG at various times $t$ for benzene molecules in porous medium, formed by random packed glass spheres with diameters of $53 \div 63 \mu \mathrm{m}$.

In the given situation, the diffusion attenuation can often be represented as a sum of exponentials

$$
\Psi(q, t)=\sum_{i} p_{i} \exp \left(-q^{2} D_{i} t\right)
$$

By representing diffusion attenuations as the sum (3) it is implied, that unknown function of the probability density $F(x, t)$ can be represented as the sum of Gaussian components $f_{i}(x, t)$ with weights $p_{i}$ :

$$
F(x, t)=\sum_{i} f_{i}(x, t) p_{i}
$$

The quantities $p_{i}$ in Eq.(4) should satisfy the obvious condition

$$
\sum_{i} p_{i}=1
$$


In the porous media for any diffusion time it is possible to choose a set of the molecules which have exactly $i$ interactions with porous walls $(i=0,1,2,3, \ldots)$. In this case, the quantity $p_{i}$ accepts the sense of a probability to find out a diffusant molecule, which has made exactly $i$ acts of interactions with the pore walls, and the functions $f_{i}(x, t)$ will represent the probability density for a diffusion displacement $x$, having exactly $i$ interactions with the walls. Within the framework of the accepted assumption, the probability $p_{0}$ and the function $f_{0}(x, t)$ will refer to those diffusant molecules, which during diffusion process, never interacted with the pore walls, while the sum

$$
\sum_{i \geq 1} f_{i}(x, t) p_{i}
$$

to those molecules, which have experienced such interactions.

The function $f_{0}(x, t)$ corresponds to the probability density function for displacements $x$ of diffusant molecules in the bulk liquid, that is

$$
f_{0}(x, t)=\frac{1}{\left(4 \pi D_{0} t\right)^{3 / 2}} \exp \left(-\frac{x^{2}}{4 D_{0} t}\right),
$$

where $D_{0}$ is bulk self-diffusion coefficient. Thus, Eq.(4) can be represented as

$$
F(x, t)=f_{0}(x, t) p_{0}+\sum_{i \geq 1} f_{i}(x, t) p_{i} .
$$

For times greater than some characteristic time $\tau_{p}$, which is defined by the media geometry, a dfiffusant molecules having made a lot of interactions with the pore wall, will be diffusing with some effective self-diffusion coefficient $D_{\infty}$ and with a corresponding probability density $f_{\infty}(x, t)$, again leading to Eq.(6), but now with $D_{0}$ replaced by $D_{\infty}$ :

$$
f_{\infty}(x, t)=\frac{1}{\left(4 \pi D_{\infty} t\right)^{3 / 2}} \exp \left(-\frac{x^{2}}{4 D_{\infty} t}\right) .
$$

Here $D_{\infty}$ is the limiting self-diffusion coefficient for the given porous medium.

It allows rewriting the expression for the probability density function $F(x, t)$ as

$$
F(x, t)=f_{0}(x, t) p_{0}(t)+f_{\infty}(x, t) \sum_{i \geq 1} p_{i}(t),
$$

where, in an explicit form, it is designated that the probability is some function of the diffusion time.

From Eq.(5) it follows

$$
\sum_{i \geq 1} p_{i}(t)=1-p_{0}(t)
$$

whence

$$
F(x, t)=f_{0}(x, t) p_{0}(t)+f_{\infty}(x, t)\left[1-p_{0}(t)\right] .
$$

With it, the time dependence of the self-diffusion coefficient in an NMR diffusion experiment can be represent as

$$
D(t)=D_{0} p_{0}(t)+D_{\infty}\left(1-p_{0}(t)\right) .
$$

An explicit form of the function $p_{0}(t)$ can be based on the assumption that diffusion displacements of the diffusant molecules in the porous medium between two consecutive interactions with the pore walls are random variables. Interactions of molecules with the wall can be considered as a Poisson flow of events with the probability to undergo $n$ interactions during moving over a distance $l$ 


$$
p_{n}=\frac{1}{n !}\left(\frac{l}{\lambda}\right)^{n} \exp \left(-\frac{l}{\lambda}\right),
$$

from whence it follows, that diffusion displacements $l$ of a diffusing molecule in a porous medium between two sequential interactions with the pore walls are random variables, which are distributed by the exponential law $\exp (-l / \lambda)$, where $\lambda$ is the mean magnitude of diffusion displacement of molecules between two sequential interactions with the pore walls.

Assuming that in the system "liquid - solid" there are no specific interactions and that the translational mobility of the diffusing molecules in the medium is characterized by the same characteristic sizes as in the bulk liquid, the size $\lambda$ can be treated as a free diffusion length, characteristic for the considered porous system.

The size of the molecular diffusion displacement $l$ in time $t$ is a random variable, defined by the density function

$$
F(l)=\frac{4 \pi l}{\sqrt{\left(4 \pi D_{0} t\right)^{3}}} \exp \left(-\frac{l^{2}}{4 D_{0} t}\right) .
$$

Then, as size $l$ in expression (13), it is possible to use the average value $\langle l\rangle$ which is defined by the expression

$$
\langle l\rangle=\frac{4 \pi}{\sqrt{\left(4 \pi D_{0} t\right)^{3}}} \int_{0}^{\infty} l^{3} \exp \left(-\frac{l^{2}}{4 D_{0} t}\right) d l=\frac{4 \sqrt{D_{0} t}}{\sqrt{\pi}} .
$$

This allows to define the probability $p_{0}(t)$ as

$$
p_{0}(t)=\exp \left(-\frac{4 \sqrt{D_{0} t}}{\sqrt{\pi} \lambda}\right) .
$$

Thus, the time dependence of the self-diffusion coefficient over the whole interval of diffusion times can be represented as

$$
D(t)=\left(D_{0}-D_{\infty}\right) \exp \left(-\frac{4 \sqrt{D_{0} t}}{\sqrt{\pi} \lambda}\right)+D_{\infty} .
$$

The size of the free diffusion length $\lambda$ can be estimated by assuming that any pore can be replaced by an (effective) spherical pore, having the same value of the surface area as a real pore.

\section{Conclusions}

As a direct consequence of Eq.(17) is the time dependence of the self-diffusion coefficient may be represented in the coordinates $\left(\frac{D(t)-D_{\infty}}{D_{0}} ; \sqrt{D_{0} t}\right)$.

As an illustration, Fig. 2 present the time dependence of the self-diffusion coefficient of xenon in a medium consisting of randomly packed glass spheres (symbols), obtained in [2]. All time dependences are linearized according to the Eq.(17). The straight line corresponds to the exponential dependence

$$
\frac{D(t)-D_{\infty}}{D_{0}}=\left(1-\frac{D_{\infty}}{D_{0}}\right) \exp \left(-\frac{7,8 \sqrt{D_{0} t}}{d}\right),
$$

where $d$ is the diameter the glass spheres. 


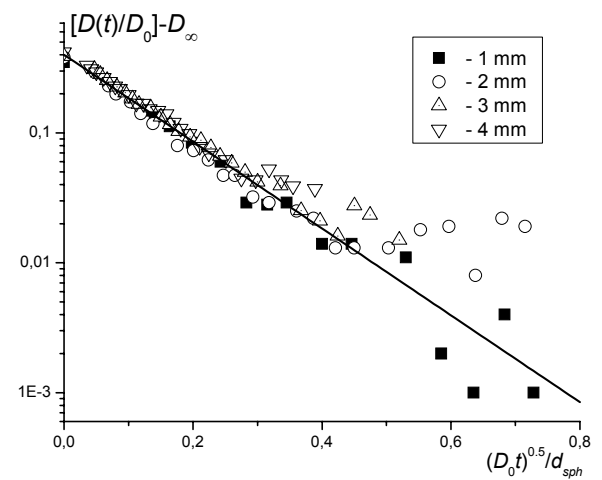

Fig. 2: Time dependence of the self-diffusion coefficient of ${ }^{129}$ Xenon in a porous medium formed by randomly packed glass spheres of various diameters $(d=1,2,3,4 \mathrm{~mm})$ [2]. All self-diffusion coefficients are normalizing by spheres diameters. The solid line corresponds to Eq.(18).

The work is supported by RFBR grant 07-02-96604-r_povoljie_a.

\section{References}

[1] Stejskal E.O., Tanner J.E. J. Chem. Phys. 42 (1965) 288.

[2] R.W. Mair, P.N. Sen, M. D. Hurlimann et al., J. Magn. Reson. 156 (2002) 202-212. 